\title{
Light-sheet microscopy by confocal line scanning of dual-Bessel beams
}

Pengfei Zhang

Mary E. Phipps

Peter M. Goodwin

James H. Werner 


\section{Light-sheet microscopy by confocal line scanning of dual-Bessel beams}

\author{
Pengfei Zhang, ${ }^{a}$ Mary E. Phipps, ${ }^{\text {b }}$ Peter M. Goodwin, ${ }^{b}$ \\ and James $H$. Werner ${ }^{b, *}$ \\ aWashington University in St. Louis, Department of Biomedical \\ Engineering, Optical Imaging Laboratory, One Brookings Drive, \\ St. Louis, Missouri 63130-4899, United States \\ ${ }^{b}$ Center for Integrated Nanotechnologies, Los Alamos National \\ Laboratory, Mail Stop G755, Los Alamos, New Mexico 87545, \\ United States
}

\begin{abstract}
We have developed a light-sheet microscope that uses confocal scanning of dual-Bessel beams for illumination. A digital micromirror device (DMD) is placed in the intermediate image plane of the objective used to collect fluorescence and is programmed with two lines of pixels in the "on" state such that the DMD functions as a spatial filter to reject the out-of-focus background generated by the side-lobes of the Bessel beams. The optical sectioning and out-of-focus background rejection capabilities of this microscope were demonstrated by imaging of fluorescently stained actin in human A431 cells. The dualBessel beam system enables twice as many photons to be detected per imaging scan, which is useful for low light applications (e.g., single-molecule localization) or imaging at high speed with a superior signal to noise. While demonstrated for two Bessel beams, this approach is scalable to a larger number of beams. () The Authors. Published by SPIE under a Creative Commons Attribution 3.0 Unported License. Distribution or reproduction of this work in whole or in part requires full attribution of the original publication, including its DOI. [DOI: 10.1117/1.JBO.21.10.100502]
\end{abstract}

Keywords: fluorescence microscopy; three-dimensional microscopy; confocal microscopy.

Paper 160533LR received Aug. 4, 2016; accepted for publication Oct. 6, 2016; published online Oct. 25, 2016.

Fluorescence microscopy is widely used for tissue and cell imaging due to its noninvasiveness and the ability to label and image specific structures. Since the discovery of the first fluorescent protein and the development of a number of genetic variants with emission spanning the entire visible spectrum, fluorescence microscopy has grown as a method for in vivo observation. ${ }^{1}$ When in vivo three-dimensional (3-D) or timelapse imaging is necessary, the resolution, potential photo-damage, and imaging speed are three major concerns, with it being difficult to address all three concerns simultaneously. While wide-field fluorescence microscopy is fast, whole cell illumination results in the loss of axial resolution and severe photo-bleaching and photo-damage. Confocal laser scanning microscopy provides optical sectioning capability through point excitation and out-of-focus rejection with a pinhole, at the cost of substantially reduced imaging speed. ${ }^{2}$ Although Nipkow spinning disk

*Address all correspondence to: James H. Werner, E-mail: jwerner@lanl.gov. microscopy increases the scanning speed and allows video-rate confocal imaging, ${ }^{3}$ the effective light dose in a confocal microscope with one-photon excitation is almost the same as widefield illumination ${ }^{2}$ and thus does nothing to help minimize photo-damage.

Light-sheet microscopy (LSM) is a relatively recent method that overcomes the three major concerns mentioned above. As the excitation volume is confined to very thin layer in the specimen, excellent optical sectioning capability and reduced photo-bleaching and photo-damage are obtained. ${ }^{4-6}$ In addition, since an entire slice of the sample is illuminated, the resulting fluorescence from a large field of view (FOV) can be collected all at once with an array-detector such as a CCD camera, yielding imaging speeds comparable to a wide-field microscope. For these reasons, LSM is growing in popularity, with applications in 3-D and single-molecule imaging. ${ }^{7-9}$ LSM was initially performed by focusing a Gaussian beam into a thin sheet of light with a cylindrical lens, an approach that works well for relatively large (millimeter) sized samples such as zebrafish. ${ }^{4}$ However, for many samples (e.g., single mammalian cells only a few microns high but tens of microns wide), the diffraction of a tightly focused Gaussian beam makes this technique difficult to implement for small beam waists (e.g., less than microns) over large FOVs (tens of microns). ${ }^{10,11}$ To circumvent issues related to diffraction, a number of laboratories have turned to scanning a propagation invariant beam, such as Bessel beam or Airy beam, for excitation in LSM, enabling diffraction-limited excitation over a large FOV. ${ }^{6,12,13}$ In addition to its favorable divergence properties in a homogenous medium, the self-healing properties of a Bessel beam allow minimal degradation of its profile when travelling in scattering media and thus provide improved optical sectioning. ${ }^{14}$

While the side-lobes of a Bessel beam play important roles in its propagation invariance and self-healing properties, these side-lobes also generate an out-of-focus background when the Bessel beam is scanned in LSM for fluorescence excitation. This unwanted background can be reduced using appropriate illumination strategies such as two-photon excitation or structural illumination, but at the expense of greater cost and complexity., ${ }^{5,6,13}$ An alternative approach is to spatially filter the out-of-focus fluorescence during detection. Just like a pinhole in a confocal point scanning system, in LSM a slit (either physical or virtual) can be used for spatial filtering of the excitation laser beam. ${ }^{11-17}$ For physical slits, descanning the moving laser beam prior to its passage through the stationary physical slit is required. ${ }^{17}$ Others have used a CMOS camera working in rolling shutter mode, with the rolling row of pixels synchronized with beam motion as a virtual slit. ${ }^{16,18}$ When the attempt is made to increase the imaging speed by scanning an array of parallel laser beams, ${ }^{19}$ it is hard to achieve confocal detection with physical slits. While dual-slit filtering is realizable with the rolling shutter of a CMOS camera, ${ }^{18}$ it is unclear how to extend this method beyond two beams.

Here, we show the use of a digital micromirror device (DMD) programmed as a slit array and used for background rejection in LSM. We note DMDs have been previously used to create pinhole arrays for parallel point scanning confocal microscopy ${ }^{20,21}$ and here, we extend the method to multiline LSM microscopy, showing a proof-of-principle experiment that scans dual-Bessel beams for cell illumination. The fluorescence from the Bessel beam excitation is projected on a DMD that is programmed to create a dual-slit, spatially filtering the 
out-of-focus background generated by the side-lobes of the Bessel beams. As compared to the single Bessel beam line-scanning system, the use of dual-Bessel beams enables twice the number of collected photons per imaging frame. Although we present the DMD slit concept with only two Bessel beams, the extension to a larger number of beams is straightforward. Using a diffractive beams splitter to create multiple Bessel beams coupled to the DMD spatial filter system one could conceivably increase the imaging speed of LSM by an order of magnitude.

A schematic diagram of our dual-Bessel beam scanning light-sheet microscope system is shown in Fig. 1. A pigtailed laser diode operating at $488 \mathrm{~nm}$ (LP488-SF20, Thorlabs) was used for excitation. The laser light coming out from the fiber was collimated by a microscope objective $(20 \times)$ and passed through an axicon with a base angle of 1 deg (Thorlabs), behind which a Bessel beam was created. ${ }^{19}$ Along its propagation direction, the Bessel beam evolved into a conical ring with constant thickness. A lens, one focal length away from the axicon, was used to create a focused ring onto a galvo-mirror (GVSM001, Thorlabs). Between this lens and the galvo-mirror, the beam is split and recombined into two beams. A pair of relay optics imaged both focused rings onto the back focal plane (BFP) of the excitation objective (EO) (CFI APO $40 \times / 0.8 \mathrm{~W}$, Nikon), and two Bessel beams were reconstructed near the focal plane of the EO. The small difference in the incidental angles of the beams onto the galvo-mirror allowed the formation of two parallel and well-separated Bessel beams behind the EO (see Fig. 2), and the separation of the dual-Bessel beams can be tuned by adjusting their incidental angles. The galvo-mirror was

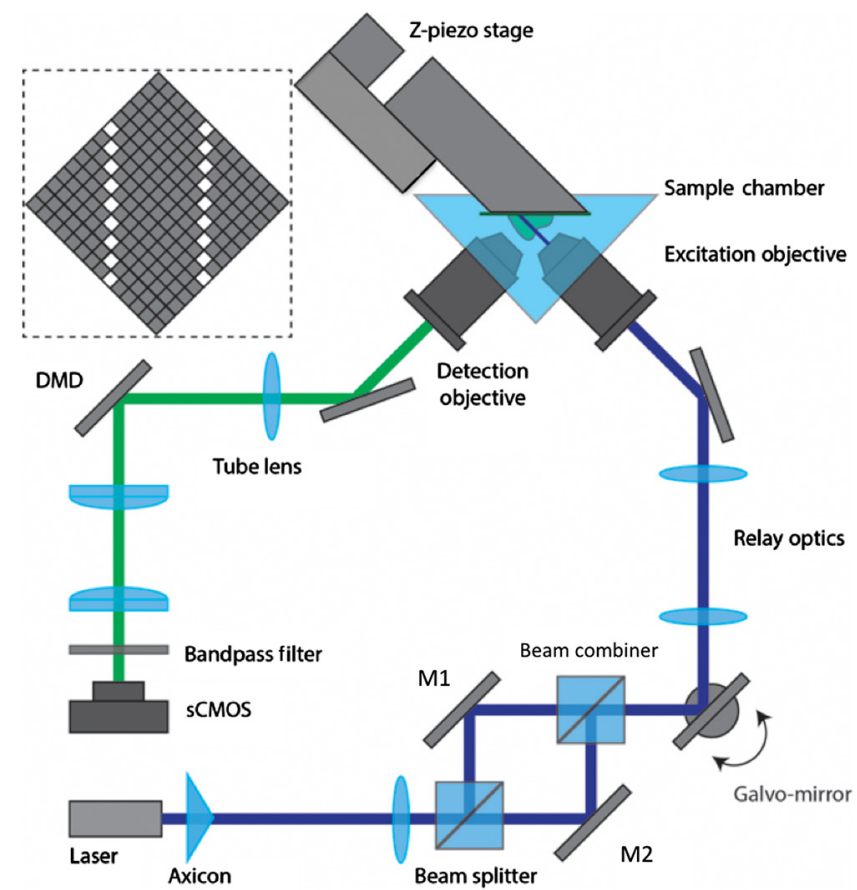

Fig. 1 A schematic diagram of the confocal dual-Bessel beam scanning light-sheet microscope. The inset shows the arrangement of the DMD device and the displayed dual-slit pattern. The two objectives are sealed into the water-filled sample chamber via rubber membranes (not shown), which keeps the chamber water-tight while allowing enough movement of the objectives for good optical alignment. The two beams are separated in a plane perpendicular to the paper and can be tuned by tilting the mirrors $\mathrm{M} 1, \mathrm{M} 2$, and the beam splitter. used to rapidly translate both Bessel beams laterally across the specimen, forming a thin excitation sheet. The fluorescence from this thin excitation layer was collected by a detection objective (DO) (CFI APO $40 \times / 0.8 \mathrm{~W}$, Nikon) oriented at a right angle to the EO such that its focal plane coincided with the excitation layer. The collected fluorescence was relayed by a tube lens to a DMD (DLI4130 high-speed kit, Digital Light Innovations). The DMD is equipped with a $1024 \times 768$ array of micrometer-sized mirrors. Each of the micromirrors can be independently tilted by \pm 12 deg along its diagonal axis, to an "on" or "off" state, with a speed as high as $22 \mathrm{kHz}$. The DMD was rotated by $45 \mathrm{deg}$ such that the fluorescence was deflected in the horizontal plane. The DMD was programmed such that only two separated vertical lines of pixels are in the "on" state (e.g., reflected towards the camera), with these lines serving as spatial filters for the two excitation Bessel beams (Fig. 2). The spatially filtered fluorescence was then imaged onto the focal plane of a scientific CMOS camera (ORCA-Flash4.0, Hamamatsu) operating in rolling shutter mode. A number of double-line patterns with different horizontal shifts matching the lateral Bessel beam locations were preloaded on the DMD, with these patterns synchronized with the galvo-mirror such that the slits follow the Bessel beams.

The specimen to be imaged was placed on a coverslip and mounted in a homemade sample holder as previously described. ${ }^{22}$ Due to the use of two long-working distance water-dipping objectives for excitation and detection, a custom water-filled V-shaped sample chamber was used to surround the sample. The sample holder was inserted into the water-filled chamber

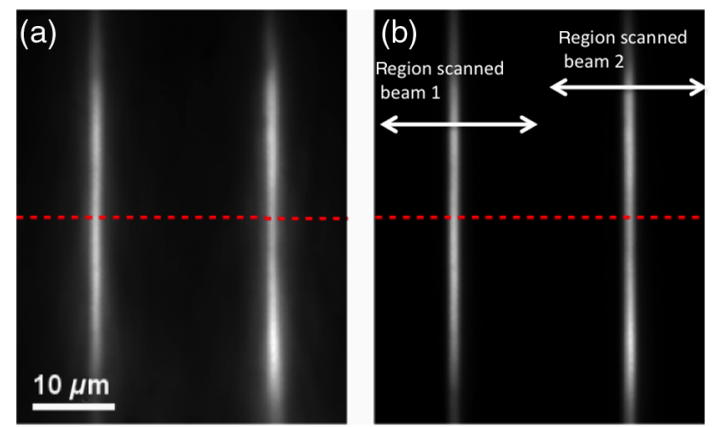

(c)

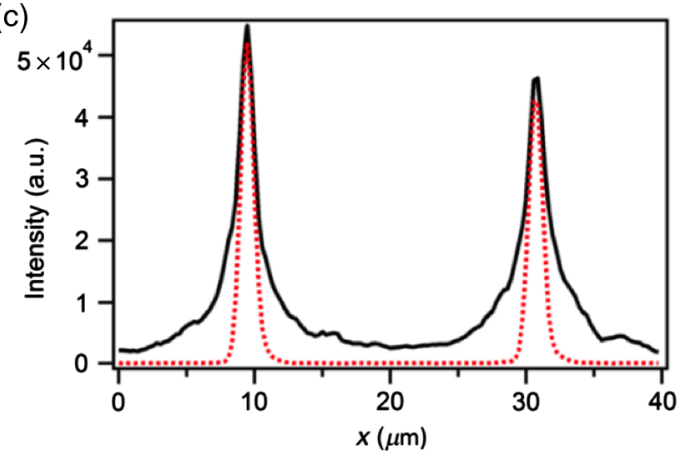

Fig. 2 Fluorescence image from dual-Bessel beam excitation. (a) Fluorescence image of quantum dots immobilized in PDMS excited by the dual-Bessel beams and taken with the DMD in mirror mode (all pixels in "on" state). (b) The same image taken with the DMD in slit mode, with the area scanned by each beam depicted by the white arrows. (c) Fluorescence distribution along the dashed lines shown in (a) and (b) for mirror mode (black solid line) and slit mode (red dotted line). 
at an angle of $45 \mathrm{deg}$ with respect to vertical, as shown in Fig. 1. The sample holder was attached to a piezo stage (Nano-F100S, Mad City Labs) that moved the sample along the optical axis of the DO such that images at different $Z$-depths could be acquired. The piezo stage and sample holder were mounted on a three-axis manual translation stage that permitted the region of interest in the specimen to be selected and brought into focus.

The spatial profiles of the Bessel beams (their central lobe diameter and longitudinal extension) are controlled by the diameter and thickness of the optical ring at the BFP of the EO. For our system, we used magnification in the relay optics to yield a Bessel beam with a central lobe diameter of $\sim 500 \mathrm{~nm}$ and a longitudinal extension of $\sim 50 \mu \mathrm{m}$, as shown in Fig. 2. The spatial profiles of the Bessel beams in the image plane were measured by examining the fluorescence from a thin layer of quantum dots [Qdot® 605 ITK $^{\mathrm{TM}}$ amino (PEG), Life Technologies] immobilized in polydimethylsiloxane (PDMS) (Sylgard 182, Dow Corning). The quantum dot fluorescence was isolated from the scattered excitation light using a bandpass filter (HQ620/60, Chroma) placed in front of the camera. To reject the unwanted fluorescence generated by the side bands of the Bessel beams and to allow the passing of the central peaks of the Bessel beams, the DMD was programmed with two lines of pixels in the "on" state, with each line acting as a slit. The slit width was programmed to be eight pixels wide, which is equivalent to $1 \mu \mathrm{m}$ in the sample plane, a size chosen to pass the central portion of the Bessel beam while rejecting the side-lobes. As shown in Fig. 2, this slit width effectively rejects the side-band fluorescence while preserving the central peak of the Bessel beam, with a resolution comparable to a single spatial filter/ single slit geometry. We note that as long as the beams are well separated (e.g., side-lobes do no overlap), this approach can be extended to a larger number of Bessel beams.

The two Bessel beams were scanned by the galvo-mirror across the focal plane of the DO for light-sheet excitation. The galvo-mirror was driven by a sawtooth voltage waveform that was generated from a data acquisition (DAQ) board, (PCIe6321, National Instruments). To synchronize the DMD patterns with the moving Bessel beams, the frame synchronization signal exported from the DMD controller board was used as the external sampling clock of the DAQ board. With a pattern display time of $64 \mu$ s (thus a sampling rate of $\sim 16 \mathrm{kHz}$ ) and a scanning step size of $320 \mathrm{~nm}, \sim 4 \mathrm{~ms}$ is required to cover a lateral scanning distance of $40 \mu \mathrm{m}$. The experimental control and data acquisition were automated with custom LabVIEW software.

As a demonstration of our microscopy system, we examined fixed human A431 cells with their actin labeled with Alexa Fluor® 488 Phalloidin. A431 cells (ATCC \#CRL-1555) were cultured in high glucose Dulbecco's modified eagle medium (ATCC \#30-2002) supplemented with $10 \%$ fetal calf serum (Hyclone) and grown at $37^{\circ} \mathrm{C}$ in $5 \% \mathrm{CO}_{2}$ at $100 \%$ humidity. To seed sterile glass coverslips, cells were trypsinized at about $80 \%$ confluency, centrifuged, and then resuspended in culture medium at about $4 \times 10^{5}$ cells $/ \mathrm{ml}$. This cell suspension was added to coverslips in a $10-\mathrm{cm}$ tissue culture plate and incubated overnight at $37^{\circ} \mathrm{C}$. The coverslips were then washed three times with phosphate-buffered saline (PBS), and fixed in $4 \%$ paraformaldehyde for $20 \mathrm{~min}$. The fixed cells were washed again with PBS, permeablized with $0.1 \%$ Triton-X-100 for $5 \mathrm{~min}$, and then blocked with $1 \%$ bovine serum albumin in PBS for $10 \mathrm{~min}$. For staining, a 200-unit/ml stock of Alexa
Fluor 488-phalloidin (Life Technologies) was diluted in 1\% bovine serum albumin in PBS to a concentration of 5units $/ \mathrm{ml}$. The fixed cells were incubated in about $2 \mathrm{ml}$ of diluted dye for 20 min in the dark. After rinsing twice with PBS and water, the coverslips were then mounted for imaging. A bandpass filter HQ535/50 (Chroma) and a longpass filter LP02-488RU-25 (Semrock) placed in front of the camera blocked scattered excitation light and transmitted the fluorescence. Figures 3(a)-3(d) show single slices of human A431 cell actin taken at axial depths of $-13.2,-6.6,6.6$, and 13.2 $\mu \mathrm{m}$ when DMD was in mirror mode (all of the pixels are "on"), while Figs. 3(e)-3(h) show the same slices with the DMD in the slit mode. The power of each excitation laser beam was $250 \mu \mathrm{W}$ when spatial filtering was employed and $50 \mu \mathrm{W}$ in mirror mode, values chosen such that the two images had comparable brightness. The exposure time was $100 \mathrm{~ms}$, enabling the laser beams to be scanned over the FOV 25 times. As can be seen in Fig. 3, better image contrast and lower backgrounds are obtained when spatial filtering with the DMD in slit mode is employed. The difference can be visualized by the fluorescence intensity profiles taken along the selected dashed lines in Figs. 3(c) and 3(g), which are shown in Fig. 3(i). With the DMD in slit mode, the features are sharper and there is less background contribution from features above and below the focal plane.

In summary, we have developed a dual-Bessel beam scanning LSM system with dual-slit confocal detection for LSM. Compared to single beam scanning, the use of dual-beams increases the number of photons per imaging scan by a factor of two. A DMD device was used to reject the out-of-focus background generated by the side-lobes of the Bessel beams. We demonstrated the background rejection capability of our system by optical sectioning of human A431 cells. While these
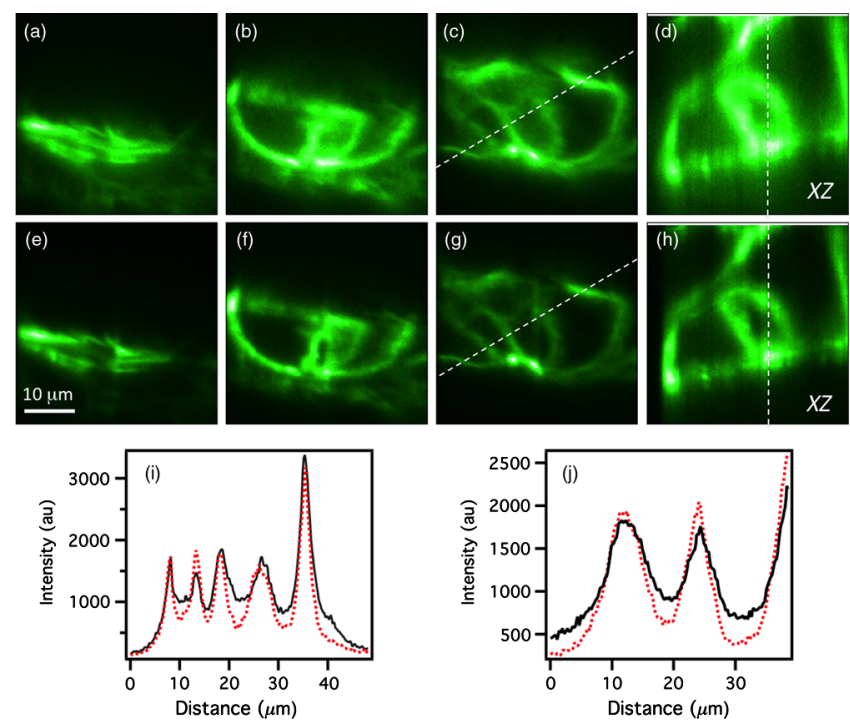

Fig. 3 Optical sectioning of human A431 cells with their actin labeled with Alexa Fluor@ 488 Phalloidin. (a-c) XY image slices taken at $Z$-depths of $-13.2,-6.6$, and $6.6 \mu \mathrm{m}$ with the DMD operating in mirror mode. (d) an $X Z$ projection taken near the central region of the $X Y$ slices shown in a-c for the DMD operating in mirror mode. (e-h) The same image slices as shown in a-d, with the DMD operating in dualslit mode. (i) Fluorescence intensity profiles along the dashed lines shown in (c), black line, and (g), red dashed line. (j) Fluorescence intensity profiles along the dashed lines shown in (d), black line, and $(\mathrm{h})$, dashed red line. All images have been scaled to enhance contrast. 
proof-of-principle experiments employed two Bessel beams and two slits on the DMD, the method can readily be extended to a larger number of Bessel beams (e.g., by diffractive optics) and slits on the DMD for even faster imaging, with an increase in the number of photons acquired per frame for a camera operating at its maximum framing rate by an order of magnitude. This has potential applications in many light-starved imaging applications, such as single-molecule-based super resolution imaging, imaging poorly transfected fluorescent cell lines, or in observing dynamic cellular processes such as cell division, bacterial infection, or microtubule extension dynamics with improved signal to noise. ${ }^{23-27}$

\section{Acknowledgments}

This work was supported through the Los Alamos Laboratory Research and Development Program and was performed at the Center for Integrated Nanotechnologies, a U.S. Department of Energy, Office of Basic Energy Sciences user facility at Los Alamos National Laboratory (Contract No. DE-AC5206NA25396).

\section{References}

1. O. Shimomura, F. H. Johnson, and Y. Saiga, "Extraction, purification and properties of aequorin, a bioluminescent protein from the luminous hydromedusan, Aequorea," J. Cell. Comp. Physiol. 59(3), 223-239 (1962).

2. J. B. Pawley, "Fundamental limits in confocal microscopy," in Handbook of Biological Confocal Microscopy, pp. 20-42, Springer, Springer, New York (2006).

3. A. Nakano, "Spinning-disk confocal microscopy. A cutting-edge tool for imaging of membrane traffic," Cell Struct. Funct. 27(5), 349-355 (2002).

4. J. Huisken et al., "Optical sectioning deep inside live embryos by selective plane illumination microscopy," Science 305(5686), 1007-1009 (2004).

5. F. O. Fahrbach et al., "Light-sheet microscopy in thick media using scanned Bessel beams and two-photon fluorescence excitation," Opt. Express 21(11), 13824-13839 (2013).

6. T. A. Planchon et al., "Rapid three-dimensional isotropic imaging of living cells using Bessel beam plane illumination," Nat. Methods 8(5), 417-423 (2011).

7. J. G. Ritter et al., "Light sheet microscopy for single molecule tracking in living tissue," PLoS One 5(7), e11639 (2010).

8. J. C. M. Gebhardt et al., "Single-molecule imaging of transcription factor binding to DNA in live mammalian cells," Nat. Methods 10(5), 421-426 (2013).

9. F. C. Zanacchi et al., "Live-cell 3D super-resolution imaging in thick biological samples," Nat. Methods 8(12), 1047-1049 (2011).
10. F. Greiss et al., "Single-molecule imaging in living drosophila embryos with reflected light-sheet microscopy," Biophys. J. 110(4), 939-946 (2016).

11. L. Gao, "Extend the field of view of selective plan illumination microscopy by tiling the excitation light sheet," Opt. Express 23(5), 61026111 (2015).

12. T. Vettenburg et al., "Light-sheet microscopy using an airy beam," Nat. Methods 11(5), 541-544 (2014).

13. O. E. Olarte et al., "Image formation by linear and nonlinear digital scanned light-sheet fluorescence microscopy with Gaussian and Bessel beam profiles," Biomed. Opt. Express 3(7), 1492-1505 (2012).

14. F. O. Fahrbach and A. Rohrbach, "Propagation stability of self-reconstructing Bessel beams enables contrast-enhanced imaging in thick media," Nat. Commun. 3, 632 (2012).

15. L. Silvestri et al., "Confocal light sheet microscopy: micron-scale neuroanatomy of the entire mouse brain," Opt. Express 20(18), 20582-20598 (2012).

16. E. Baumgart and U. Kubitscheck, "Scanned light sheet microscopy with confocal slit detection," Opt. Express 20(19), 21805-21814 (2012).

17. P. Zhang et al., "Confocal line scanning of a Bessel beam for fast 3D Imaging," Opt. Lett. 39(12), 3682-3685 (2014).

18. Z. Yang et al., "Dual-slit confocal light sheet microscopy for in vivo whole-brain imaging of zebrafish," Biomed. Opt. Express 6(5), 1797-1811 (2015).

19. L. Gao et al., "Noninvasive imaging beyond the diffraction limit of 3D dynamics in thickly fluorescent specimens," Cell 151(6), 1370-1385 (2012).

20. F. P. Martial and N. A. Hartell, "Programmable illumination and high-speed, multi-wavelength, confocal microscopy using a digital micromirror,' PLoS One 7(8), e43942 (2012).

21. A. G. York et al., "Resolution doubling in live, multicellular organisms via multifocal structured illumination microscopy," Nat. Methods 9(7), 749-754 (2012).

22. P. Zhang, P. M. Goodwin, and J. H. Werner, "Fast, 3D imaging via confocal line scanning of a Bessel beam using a single galvo mirror," Proc. SPIE 8947, 89471K (2014).

23. E. Betzig et al., "Imaging intracellular fluorescent proteins at nanometer resolution," Science 313(5793), 1642-1645 (2006).

24. M. J. Rust, M. Bates, and X. Zhuang, "Sub-diffraction-limit imaging by stochastic optical reconstruction microscopy (STORM)," Nat. Methods 3(10), 793-796 (2006).

25. S. T. Hess, T. P. Girirajan, and M. D. Mason, "Ultra-high resolution imaging by fluorescence photoactivation localization microscopy," Biophys. J. 91(11), 4258-4272 (2006).

26. J. J. Han et al., "Actin restructuring during Salmonella typhimurium infection investigated by confocal and super-resolution microscopy," J. Biomed. Opt. 19(1), 016011 (2014).

27. B.-C. Chen et al., "Lattice light-sheet microscopy: imaging molecules to embryos at high spatiotemporal resolution," Science 346(6208), 1257998 (2014) 